\title{
How to Define Cardiac Resynchronization Therapy Response
}

\author{
GERY TOMASSONI, MD, FACC, FHRS
}

\author{
Division of Electrophysiology, Baptist Health Lexington, Lexington, $K Y$
}

\begin{abstract}
Cardiac resynchronization therapy (CRT) improves quality of life, exercise capacity, and cardiac function in a significant number of patients with heart failure (HF) and left bundle branch block. CRT also reduces HF hospitalizations and overall mortality. A substantial number of patients however do not have a favorable clinical response. Evaluation of CRT non-response has been challenging as the primary mechanism for CRT improvement remains elusive. In addition, reported CRT response rates have varied significantly and are highly dependent on the criteria used to define the response. Futhermore, a disconnection exists between applying the outcome data from large clinical trials to the "real world" expectations of daily clinical practice. The ultimate goals of CRT should both met the patients' expectations for symptomatic improvement and also improve cardiac function and/or outcomes.
\end{abstract}

KEYWORDS. Response rate, cardiac resynchronization therapy, heart failure.
ISSN 2156-3977 (print) ISSN 2156-3993 (online)

(C) 2016 Innovations in Cardiac Rhythm Management

\section{Introduction}

A significant subset of patients with heart failure (HF) has cardiac dyssynchrony and delayed mechanical activation of the lateral left ventricular (LV) wall compared to the septum. Cardiac resynchronization therapy (CRT) works by improving the timing between the two segments and enhancing the overall coordination of the left ventricle. The present day approved indication for CRT is in moderate to severe HF patients with New York Heart Association class III/IV disease who are already on optimal drug therapy with LV ejection fraction $<35 \%$ in sinus rhythm and QRS width $>120 \mathrm{~ms}^{1}$

Generally speaking, CRT has been one of the most successful HF treatments to date. CRT devices have been implanted in hundreds of thousands of patients worldwide, constituting approximately $30 \%$ of symptomatic HF patients. The majority of CRT patients, approximately $70 \%$, have experienced significant improvement in both functional capacity and survival. Unfortunately, a large percentage of patients that receive CRT do not improve clinically.

Dr. Tomassoni is an advisor, speaker, and medical device board member for St. Jude Medical, Topera, Biosense Webster, Biotronics, and Medtronic; is an advisor and speaker for and has received research grants from Stereotaxis and Siemens; and is an advisor and speaker for Guidant.

Manuscript received February 29, 2016, Final version accepted April 26, 2016.

Address correspondence to: Gery Tomassoni, MD, FACC, FHRS, 1720 Nicholasville Road, Suite 601, Lexington, KY 40503. E-mail: gtomassoni@lexingtoncardio.com

\section{Qualitative Evaluation of Clinical Trials}

The results of the first CRT trials, which included MIRACLE, MUSTIC SR, MIRACLE ICD, and CONTAK-CD, ${ }^{2-5}$ demonstrated that CRT improves exercise capacity in this patient population. In comparison to the control patients, the patients who received CRT in the majority of the trials had significant improvements in the 6-minute walk test and a significant improvement in peak $\mathrm{VO}_{2}$ exchange as well.

Not only does CRT improve exercise capacity, but it can also improve cardiac function. When MIRACLE, MIRACLE ICD, and CONTAK-CD ${ }^{2,4,5}$ are compared, patients who received CRT in 2 of the 3 trials had a significant increase in LV ejection fraction (EF). In the MIRACLE trial, patients who received CRT also experienced a substantial reduction in mitral regurgitation (MR).

Morbidity and mortality were evaluated in multiple randomized, controlled trials. ${ }^{4-9}$ The pivotal Comparison of Medical Therapy and Defibrillation in Heart Failure (COMPANION) trial $^{9}$ compared optimal medical therapy versus biventricular $(\mathrm{BiV})$ pacing alone versus $\mathrm{BiV}$ pacing with implantable defibrillators (ICD) in 1,520 patients. The trial showed a significant reduction in death and/or any hospitalization with a higher eventfree survival for both CRT pacers and CRT defibrillators. The 12-month event rate reduction was approximately $19 \%$ for both groups.

In the Cardiac Resynchronization-Heart Failure (CAREHF) trial, ${ }^{8}$ all morbidity and mortality categories were significantly reduced in patients who received CRT alone without a defibrillator. The patients who received CRT had 


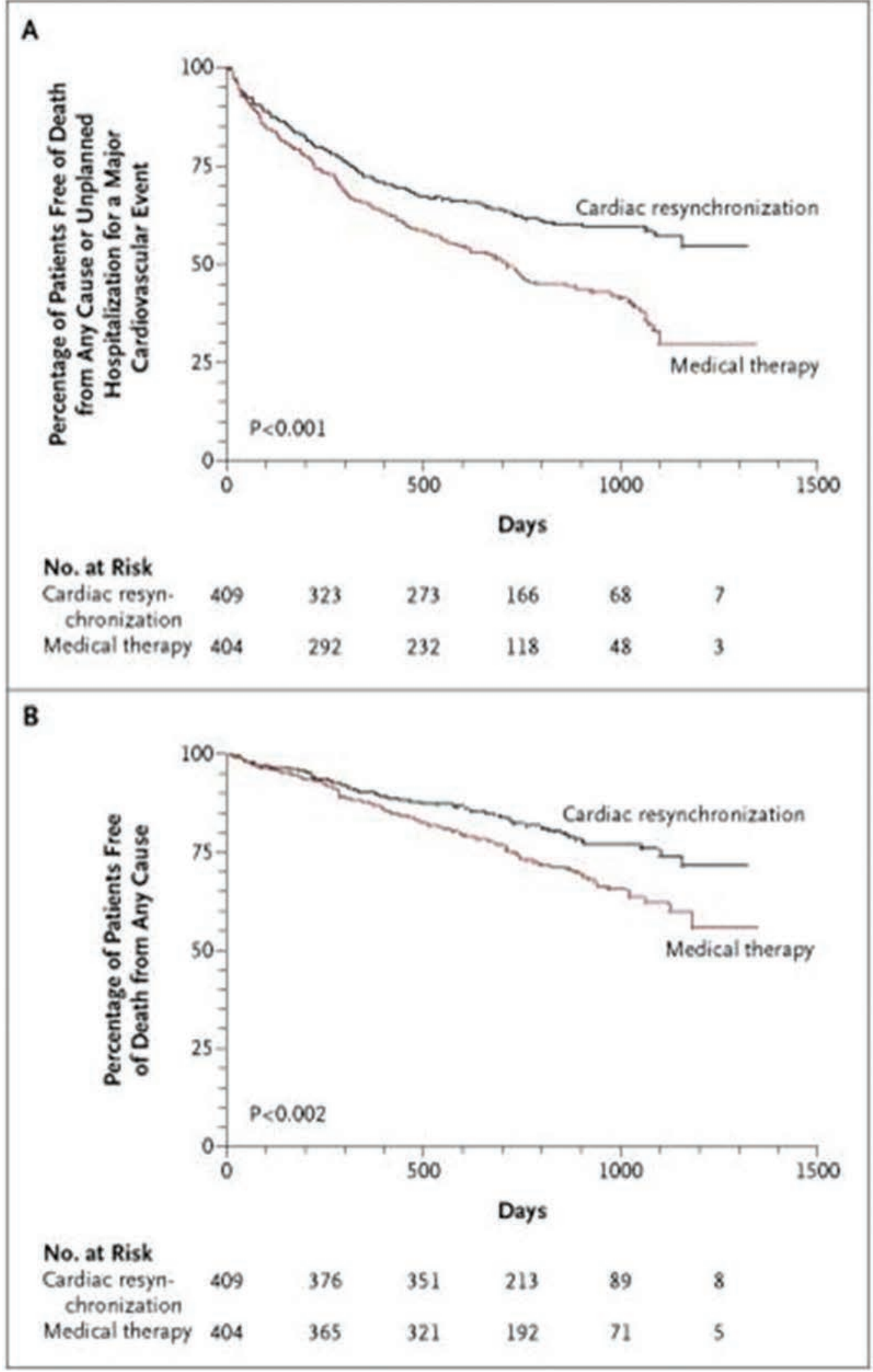

Figure 1: All-cause mortality data from CARE-HF comparing CRT (BiV) pacer alone to optimal medical therapy. From New England Journal of Medicine, Cleland JG, Daubert JC, Erdmann E, et al; Cardiac Resyn-chronization-Heart Failure (CARE-HF) Study Investigators, The effect of cardiac resynchronization on morbidity and mortality in heart failure, 352(15), 1544, Copyright (c) (2005), Massachusetts Medical Society. Reprinted with permission from Massachusetts Medical Society. 


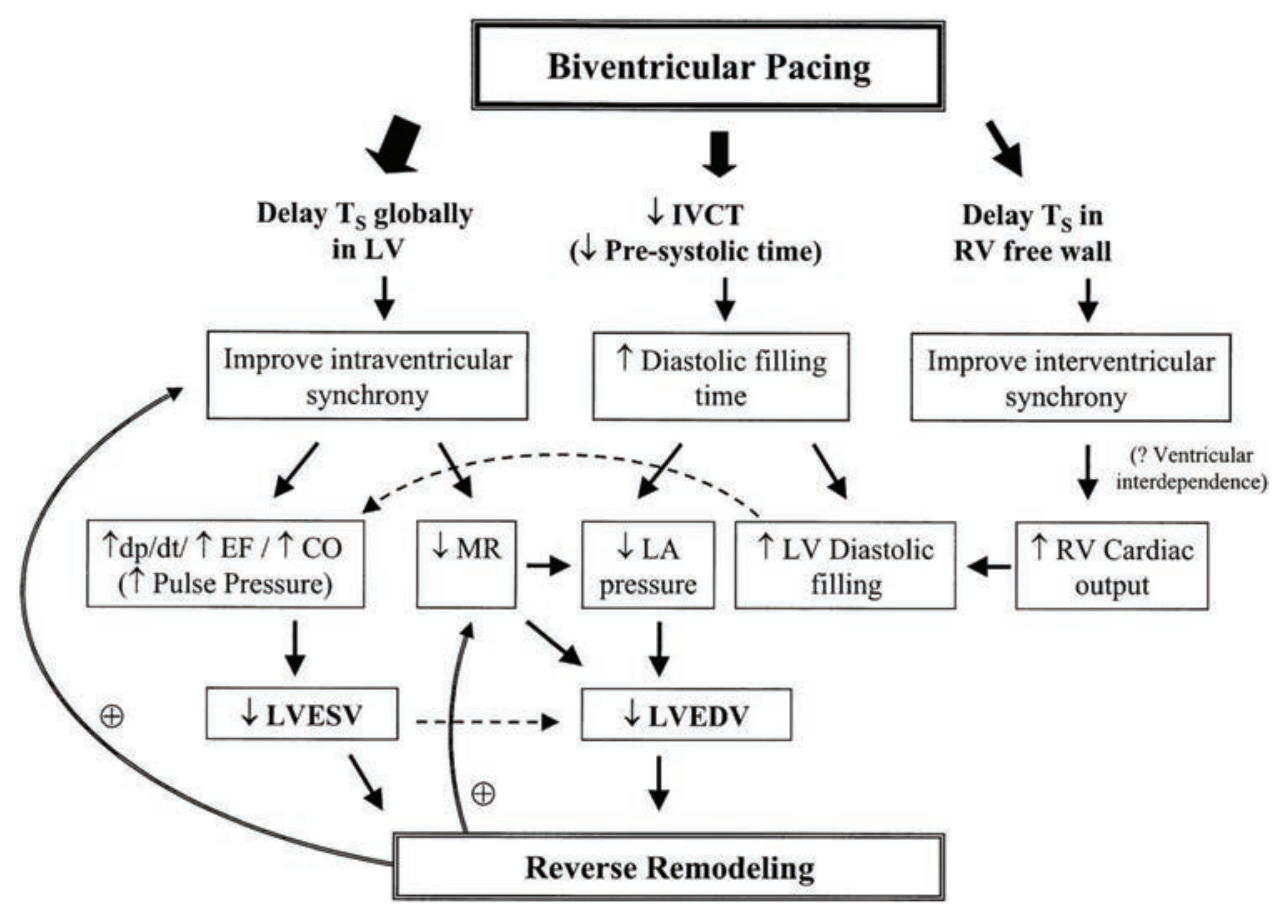

Figure 2: Cardiac resynchronization: proposed mechanisms. From Circulation, Yu CM, Chau E, Sanderson JE, et al, Tissue Doppler Echocardiographic Evidence of Reverse Remodeling and Improved Synchronicity by Simultaneously Delaying Regional Contraction After Biventricular Pacing Therapy in Heart Failure, 105(4), 444, Copyright (c) (2002), American Heart Association. Reprinted with permission from Wolters Kluwer Health, Inc.

significantly improved event-free survival compared to those who received optimal medical therapy. The two survival curves started to separate at approximately 6 months and then persisted throughout the entire duration of the trial, suggesting that the CRT response was a sustained response (Figure 1). In addition, the all-cause mortality included both reduction in sudden cardiac death and reduction in death due to worsening HF.

\section{CRT Response}

\section{Mechanisms that lead to LV Reverse Remodeling}

Figure 2 illustrates the theory behind cardiac resynchronization. ${ }^{10}$ CRT can improve intraventricular synchrony, atrioventricular synchrony, and interventricular synchrony.

Intraventricular synchrony. As a result of improved synchrony within the LV ventricle, systole becomes more effective, and therefore LV EF, cardiac output (CO), and other parameters of cardiac function are improved. Both LV end-systolic volume (LVESV) and MR (attributable to distortion of the mitral apparatus) are reduced. Subsequently, lowering of the left atrial (LA) pressure and LV end-diastolic volume (LVEDV) occurs.

Atrioventricular synchrony. A second mechanism is the shortening of the isovolumic contraction time (IVCT) after optimization of the atrioventricular (AV) delay. The effective diastolic filling time is increased, which in turn raises the stroke volume. In addition, LA pressure is reduced due to decreases in presystolic MR.

Interventricular synchrony. A less important mechanism is the improvement of interventricular synchrony between the right and left ventricles. This benefit may mediate through ventricular interdependence. This results in the gain in right ventricular (RV) $\mathrm{CO}$, thereby augmenting $\mathrm{LV}$ filling, and resulting in overall improved cardiac function. The end effect of reverse remodeling will additionally improve cardiac synchrony and decrease secondary MR, forming a positive feedback loop.

In the study by $\mathrm{Yu}$ et $\mathrm{al}_{1}^{10}$ withholding $\mathrm{BiV}$ pacing resulted in loss of cardiac benefits. Improvements in diastolic filling time, IVCT, and myocardial performance index (MPI) were lost immediately, since they were largely dependent upon control of AV synchrony. Benefits in EF, CO, quality of life, and walking distance were gradually lost over 4 weeks, which suggests that $\mathrm{BiV}$ pacing may reverse $\mathrm{LV}$ remodeling.

\section{Electrical versus Mechanical Dyssynchrony}

Two categories of dyssynchrony have been described: electrical and mechanical. With electrical dyssynchrony, there is abnormal conduction between the atria and the ventricles, between the RV and $\mathrm{LV}$, and, more importantly, within the LV. Mechanical dyssynchrony is caused by abnormal wall motion due to increased cardiac workload and stress. This involves the presence of scar and many other factors, including disruption of myocardial collagen matrix. Both of these mechanisms result in a negative impact on cardiac filling, contractility, and CO. 
Table 1: Clinical studies: QRS width inclusion criteria. ${ }^{4,5,11-17}$

\begin{tabular}{lll}
\hline QRS $\geq 120 \mathrm{~ms}$ & QRS $\geq \mathbf{1 3 0} \mathrm{ms}$ & QRS $\geq \mathbf{1 5 0} \mathbf{~ m s}$ \\
\hline CONTAK-CD & MIRACLE & MUSTIC \\
COMPANION & MIRACLE ICD & PACMAN \\
PATH-CHF I \& II & InSync III & CARE-HF \\
CARE-HF* (+ echo) & & \\
\hline
\end{tabular}

The simplest way to measure electrical dyssynchrony is the QRS width. Table 1 presents several HF studies that demonstrated the chronic benefit of CRT and the associated QRS width criterion. ${ }^{4,5,11-17}$ Patients with QRS widths of $120-150 \mathrm{~ms}$ have also been studied. ${ }^{4,5,11-17}$ CARE-HF ${ }^{14}$ used echocardiographic determination to assess mechanical dyssynchrony for patients with QRS widths between $120 \mathrm{~ms}$ and $150 \mathrm{~ms}$.

A reduction in intraventricular dyssynchrony has been shown to predict CRT response. ${ }^{18}$ QRS width has been shown to correlate well with interventricular dyssynchrony $^{19}$ but unfortunately has poor accuracy for detecting intraventricular dyssynchrony. ${ }^{20}$ As a result, it is estimated that only $70 \%$ of patients with left bundle branch block (LBBB) have echocardiographic evidence of mechanical dyssynchrony. ${ }^{20}$

$\mathrm{Yu}$ and colleagues ${ }^{21}$ also demonstrated that the QRS duration may not be a reliable predictor of mechanical dyssynchrony in HF. The study assessed mechanical dyssynchrony in control patients and HF patients stratified by QRS duration. Mechanical dyssynchrony was assessed using echocardiography and was defined as the standard deviation in the time to peak systolic contraction in $12 \mathrm{LV}$ segments. The study found that a majority of $\mathrm{HF}$ patients exhibit systolic mechanical dyssynchrony, including nearly $50 \%$ of $\mathrm{HF}$ patients with a "narrow" QRS ( $<120 \mathrm{~ms})$. Similar to previous studies $^{20}$ only $70 \%$ of patients with a wide QRS (>120 ms) had mechanical dyssynchrony present.

The same group of investigators ${ }^{22}$ then examined baseline mechanical dyssynchrony to determine if it would predict CRT response. To illustrate the importance of patient selection based on mechanical synchrony, Yu and colleagues assessed baseline ventricular dyssynchrony and response to CRT as measured by reverse remodeling (reduction in LV volume) in HF patients. The study found that those HF patients with greater baseline systolic mechanical dyssynchrony exhibited greater reverse remodeling after 3 months of CRT ("responders"). The "non-responders" did not have significant baseline mechanical asynchrony and therefore did not reach the same results in reverse remodeling.

\section{Best Measurement or Imaging Tool for Dyssynchrony}

For electrical dyssynchrony, QRS width and morphology are important predictors of CRT response, particularly when QRS is $>150 \mathrm{~ms}$ in patients with LBBB. However, patients with right bundle branch block (RBBB) or RV paced complexes do not respond as well. Another measurement of LV electrical delay is known as QLV, which is the interval that is measured from the onset of the surface QRS to the first large positive or negative peak of the LV electrogram. Studies have shown that QLV can be a strong predictor of CRT response. ${ }^{23}$

For mechanical dyssynchrony, the echocardiographic measurements or tools that are presently being evaluated include LV strain and speckled tissue tracking analysis, as well as three-dimensional volumeters. In the nonechocardiographic measurements and imaging tools, cardiac MRI, CCTA, and $\mathrm{dP} / \mathrm{dt}_{\max }$ have also been studied. However, significant controversy exists over the most ideal measurement.

\section{Definition of CRT response}

Table 2 shows the three categories of CRT response definitions. The first is based on clinical measures (patient symptoms and functional assessment). New York Heart Association class and quality of life measurements, in addition to the 6-minute walk test, exercise duration, and metabolic exercise tests, are typical clinical measures. The second category is based on LV reverse remodeling assessment. This can be performed either in the acute stage during CRT implantation and is assessed by hemodynamic parameters such as $\mathrm{CO}$, or in the chronic stage assessed by an increase in LVEF or a decrease in LV end systolic/diastolic volumes and MR. The final category includes outcome measures assessment. The measures are reductions in HF hospitalization, morbidity, and all-cause mortality. ${ }^{24}$ These primary event-driven endpoints are used in large clinical trials to define CRT response. Secondary endpoints usually assess both cardiac function and functional status. However, the adoption of the results from large clinical trials in daily practice has been difficult. For example, it would be difficult to adopt the endpoint of mortality for assessing CRT response in an individual patient, as there is a lack of before-and-after comparison. As a result, recent clinical trials have switched to a clinical composite score to include clinical, remodeling, and outcome measures.

\section{Current Issues with CRT Response}

There are a significant number of current issues that exist when assessing CRT response. Firstly, the CRT response definition is highly dependent on the criteria used to define the response. Studies have suggested that the response rate will vary from $32 \%$ to $91 \%$, depending on the criteria that were used. Thus response rates tend to be higher when clinical measures, such as subjective

Table 2: Three categories of CRT response definitions.

\begin{tabular}{l}
\hline CRT RESPONSE DEFINITIONS \\
\hline Clinical Measures Assessment \\
NYHA class \& Quality of Life \\
6 min walk test, exercise duration, \& metabolic exercise \\
tests (CPX) \\
LV Reverse Remodeling Assessment \\
Acute: Hemodynamic parameters (C.O., LV dP/dt ${ }_{\text {max }}$ ) \\
Chronic: Increase in LVEF, reduction in LV end systolic/ \\
diastolic volumes \& MR \\
Outcome Measures Assessment \\
Reductions in HF hospitalizations, morbidity, \& all cause \\
mortality
\end{tabular}


measurements, are used but are much lower when remodeling or outcome measurements are used. Also in clinical trials, there is no consensus on the optimal timeline to assess response.

Secondly, response criteria may vary greatly among investigators. For example, symptomatic improvement does not always correlate with improvement in echo or functional assessment parameters, and vice versa. Complicating factors also include the fact that acute hemodynamic or echocardiographic parameters have not been associated consistently with long-term clinical response. In addition, the best criteria to determine CRT response are unknown, and there is no true agreed surrogate for mechanical dyssynchrony.

Finally, multiple different factors between individual patients can affect the response. These factors include genetic and sex differences, stage and cause of congestive HF, LV lead location, QRS morphology and width, the presence of multiple comorbidities, LV scar in ischemic patients, and the frequency of atrial fibrillation and/or premature ventricular contractions. Device management, including optimizing $\mathrm{AV} / \mathrm{VV}$ intervals and programming to ensure the greatest percentage of $\mathrm{BiV}$ pacing is another important consideration.

Newer trials have shown that women generally have higher rates of CRT response. Severely remodeled LV can be "beyond repair," and these patients are less likely to respond to CRT. ${ }^{25}$ Two predictors of adverse remodeling and poorer clinical outcomes are severe LV dilatation and MR. ${ }^{26,27}$ Non-ischemic patients generally have better outcomes compared to ischemic patients.

Regarding atrial fibrillation in HF, approximately $25 \%$ of patients with $\mathrm{HF}$ and up to $50 \%$ of patients with class IV HF will have atrial fibrillation. In a MADIT-CRT subgroup analysis of 213 patients who had atrial fibrillation, the investigators found that CRT had less effect on outcomes in patients with atrial fibrillation. ${ }^{28}$ Patients with atrial fibrillation had poorer outcomes with higher all-cause mortality. In a meta-analysis involving 1,164 patients with CRT and atrial fibrillation, despite overall clinical improvement, the benefits appeared to be smaller compared to those who were in sinus rhythm. $^{29}$

Recently, the MADIT-CRT trial showed that LV lead location matters. Apical placement of the LV lead may enhance lead stability but is associated with worst outcomes. ${ }^{30,31}$ In the trial, distal LV lead placement increased the risk of death and/or HF hospitalization by a factor of 1.64 and increased the risk of mortality by 2.6. Therefore, LV basal pacing was better. Other trials have shown that pacing at sites of late LV activation can also improve outcomes. ${ }^{23}$

QRS width, as discussed earlier, can be an important predictor of outcome. Patients with a wide QRS ( $>150$ ms) have the highest likelihood of responding to CRT. $^{28,32,33}$ In COMPANION, the primary endpoint was met only when the QRS was $>148$ ms. In REVERSE, the primary endpoint was met when QRS was $>152 \mathrm{~ms}$. In MADIT-CRT, there was a $41 \%$ reduction in HF in patients with a QRS of $>150 \mathrm{~ms}$. With respect to QRS width of $120-150 \mathrm{~ms}$, the results of CARE-HF ${ }^{14}$ suggest that an echo dyssynchrony evaluation can be potentially valuable. However, as previously mentioned, no reproducible single echo dyssynchrony parameter is predictive of CRT response to date.

In addition to QRS width, QRS morphology is also important. Patients with LBBB tend to respond better symptomatically than those with RV paced complexes or RBBB. Also, patients with LBBB have less chance of requiring a heart transplant or implantation of an $\mathrm{LV}$ system device compared to RBBB or RV paced patient populations. $^{34}$

\section{Patient Evaluation: Clinical Trials versus the Real World}

When comparing how patients are evaluated in clinical trials versus in the real world, several questions arise. Can large clinical trial data be applied to real world experience? For example, the older age groups are poorly represented in large CRT trials (average age 60), as opposed to in daily practice where a large percentage of patients who receive CRT devices are much older. Also, in real world experience, women are less likely to receive CRT devices compared to men, despite data that show that women tend to have a better response. Does it matter that hard endpoints like those in clinical trials are not met in daily practice? Should the goal of CRT be to reduce HF hospitalization even if the patient does not improve symptomatically? Can "no improvement" in a patient's clinical status be good enough in a very progressive and debilitating disease? The use of event-driven measures is appropriate in large, long-term clinical trials but may not be as meaningful in the determination of an individual's response in daily practice. In the real world, the patient's overall sense of well-being may be a more relevant measure of CRT response.

\section{Goals of CRT}

What should be our goals for CRT? This is a difficult question to answer for many reasons. In dealing with congestive HF patients, it is difficult to predict the natural history because, many times, they follow a highly variable and progressive course. In addition, it is unknown which CRT response definition - improvement in clinical symptoms or LV reverse modeling - will result in overall improved survival. Ideally, CRT response should include both a clinical assessment and a cardiac function or outcome assessment.

Hence, reasonable goals may include, firstly, to improve the patient's symptomatic status, such as decreasing the New York Heart Association class, and to meet the patient's expectations of feeling better; and secondly, to attenuate pathological LV remodeling such as via a decrease in LV end systolic/diastolic volumes or increase in LV EF.

\section{Conclusion}

CRT improves quality of life, exercise capacity, and cardiac function in a significant number of patients with HF and LBBB. Furthermore, data show that it reduces HF hospitalizations and overall mortality. The mechanisms 
for CRT are not known precisely, but reverse LV remodeling occurs by restoring cardiac synchrony. There is no single universally accepted surrogate for mechanical dyssynchrony. CRT response rate depends highly on the criteria used to define a response, and the criteria vary dramatically between clinical trials, so it is difficult to compare these trials with one another. A patient in one trial may be classified as a responder and that same patient may be a non-responder in another trial. CRT nonresponse can be attributed to multiple factors. Female gender, QRS width >150 ms, LBBB morphology, and a non-ischemic etiology demonstrate the greatest benefit to CRT. There is a significant disconnection between the clinical trial data and real world expectations, but generally speaking, in the real world, patients' overall well-being may be a more relevant measure of CRT response. Finally, the goal of CRT response should be to improve patient symptoms and reduce LV remodeling.

\section{References}

1. Epstein AE, DiMarco JP, Ellenbogen KA, et al. ACC/AHA/ HRS 2008 guidelines for device-based therapy of cardiac rhythm abnormalities: a report of the American College of Cardiology/American Heart Association Task Force on Practice Guidelines (Writing Committee to Revise the ACC/AHA/NASPE 2002 Guideline Update for Implantation of Cardiac Pacemakers and Antiarrhythmic Devices). Circulation. 2008;117(21):e350-408.

2. St John Sutton MG, Plappert T, Abraham WT, et al. Effect of cardiac resynchronization therapy on left ventricular size and function in chronic heart failure. Circulation. 2003; 107(15):1985-1990.

3. Cazeau S, Leclercq C, Lavergne T, et al. Effects of multisite biventricular pacing in patients with heart failure and intraventricular conduction delay. N Engl J Med. 2001; 344(12):873-880.

4. Young JB, Abraham WT, Smith AL, et al. Combined cardiac resynchronization and implantable cardioversion defibrillation in advanced chronic heart failure: the MIRACLE ICD Trial. JAMA. 2003;289(20):2685-2694.

5. Higgins SL, Hummel JD, Niazi IK, et al. Cardiac resynchronization therapy for the treatment of heart failure in patients with intraventricular conduction delay and malignant ventricular tachyarrhythmias. J Am Coll Cardiol. 2003;42(8):1454-1459.

6. Abraham WT, Fisher WG, Smith AL, et al. Multicenter InSync Randomized Clinical Evaluation. Cardiac resynchronization in chronic heart failure. N Engl J Med. 2002; 346(24):1845-1853.

7. Bradley DJ, Bradley EA, Baughman KL, et al. Cardiac resynchronization and death from progressive heart failure: a meta-analysis of randomized controlled trials. JAMA. 2003;289(6):730-740.

8. Cleland JG, Daubert JC, Erdmann E, et al; Cardiac Resyn-chronization-Heart Failure (CARE-HF) Study Investigators. The effect of cardiac resynchronization on morbidity and mortality in heart failure. $N$ Engl J Med. 2005;352(15):1539-1549.

9. Lindenfeld J, Feldman AM, Saxon L, et al. Effects of cardiac resynchronization therapy with or without a defibrillator on survival and hospitalizations in patients with New York Heart Association class IV heart failure. Circulation. 2007; 115(2):204-212.
10. Yu CM, Chau E, Sanderson JE, et al. Tissue Doppler echo-cardiographic evidence of reverse remodeling and improved synchronicity by simultaneously delaying regional contraction after biventricular pacing therapy in heart failure. Circulation. 2002;105(4):438-445.

11. Bristow MR, Feldman AM, Saxon LA. Heart failure management using implantable devices for ventricular resynchronization: Comparison of Medical Therapy, Pacing, and Defibrillation in Chronic Heart Failure (COMPANION) trial. COMPANION Steering Committee and COMPANION Clinical Investigators. J Card Fail. 2000;6(3):276-285.

12. Auricchio A, Stellbrink C, Sack S, et al. The Pacing Therapies for Congestive Heart Failure (PATH-CHF) study: rationale, design, and endpoints of a prospective randomized multicenter study. Am J Cardiol. 1999; 83(5B):130D-135D.

13. Stellbrink C, Auricchio A, Butter C, et al. Pacing Therapies in Congestive Heart Failure II study. Am J Cardiol. 2000; 86(9A):138K-143K.

14. Cleland JG, Daubert JC, Erdmann E, et al. The CARE-HF study (CArdiac REsynchronisation in Heart Failure study): rationale, design and end-points. Eur J Heart Fail. 2001; 3(4):481-489.

15. Abraham WT. Rationale and design of a randomized clinical trial to assess the safety and efficacy of cardiac resynchronization therapy in patients with advanced heart failure: the Multicenter InSync Randomized Clinical Evaluation (MIRACLE). J Card Fail. 2000;6(4):369-380.

16. Linde $C$, Leclercq $C$, Rex $S$, et al. Long-term benefits of biventricular pacing in congestive heart failure: results from the MUltisite STimulation in cardiomyopathy (MUSTIC) study. J Am Coll Cardiol. 2002;40(1):111-118.

17. Leclercq C, Victor F, Alonso C, et al. Comparative effects of permanent biventricular pacing for refractory heart failure in patients with stable sinus rhythm or chronic atrial fibrillation. Am J Cardiol. 2000;85(9):1154-1156, A9.

18. Bax JJ, Abraham T, Barold SS, et al. Cardiac resynchronization therapy: Part 1-issues before device implantation. J Am Coll Cardiol. 2005;46(12):2153-2167.

19. Ghio S, Constantin C, Klersy C, et al. Interventricular and intraventricular dyssynchrony are common in heart failure patients, regardless of QRS duration. Eur Heart J. 2004; 25(7):571-578.

20. Bleeker GB, Schalij MJ, Molhoek SG, et al. Relationship between QRS duration and left ventricular dyssynchrony in patients with end-stage heart failure. J Cardiovasc Electrophysiol. 2004;15(5):544-549.

21. Yu CM, Lin $H$, Zhang $Q$, Sanderson JE. High prevalence of left ventricular systolic and diastolic asynchrony in patients with congestive heart failure and normal QRS duration. Heart. 2003;89(1):54-60.

22. Yu CM, Fung WH, Lin H, Zhang $Q$, Sanderson JE, Lau CP. Predictors of left ventricular reverse remodeling after cardiac resynchronization therapy for heart failure secondary to idiopathic dilated or ischemic cardiomyopathy. Am J Cardiol. 2003;91(6):684-688.

23. Gold MR, Birgersdotter-Green U, Singh JP, et al. The relationship between ventricular electrical delay and left ventricular remodeling with cardiac resynchronization therapy. Eur Heart J. 2011;32(20):2516-2524.

24. European Heart Rhythm Association (EHRA); European Society of Cardiology (ESC); Heart Rhythm Society; et al. 2012. Expert consensus statement on cardiac resynchronization therapy in heart failure: implant and follow-up recommendations and management. Europace. 2012;14(9):1236-1286. 
25. De Boeck BW, Meine M, Leenders GE, et al. Practical and conceptual limitations of tissue Doppler imaging to predict reverse remodeling in cardiac resynchronization therapy. Eur J Heart Fail. 2008;10(3):281-290.

26. Achilli A, Peraldo C, Sassara M, et al. Prediction of response to cardiac resynchronization therapy: the selection of candidates for CRT (SCART) study. Pacing Clin Electrophysiol. 2006;29(Suppl 2):S11-19.

27. Gradaus R, Stuckenborg V, Lo«her A, et al. Diastolic filling pattern and left ventricular diameter predict response and prognosis after cardiac resynchronization therapy. Heart. 2008;94(8):1026-1031.

28. Moss AJ, Hall WJ, Cannom DS, et al. Cardiac-resynchronization therapy for the prevention of heart-failure events. N Engl J Med. 2009;361(14):1329-1338.

29. Upadhyay GA, Choudhry NK, Auricchio A, Ruskin J, Singh JP. Cardiac resynchronization in patients with atrial fibrillation: a meta-analysis of prospective cohort studies. J Am Coll Cardiol. 2008;52(15):1239-1246.
30. Singh JP, Klein HU, Huang DT, et al. Left ventricular lead position and clinical outcome in the multicenter automatic defibrillator implantation trial-cardiac resynchronization therapy (MADIT-CRT) trial. Circulation. 2011;123(11):1159-1166.31.

31. Merchant FM, Heist EK, McCarty D, et al. Impact of segmental left ventricle lead position on cardiac resynchronization therapy outcomes. Heart Rhythm. 2010;7(5):639-644.

32. Bristow MR, Saxon LA, Boehmer J, et al. Cardiac-resynchronization therapy with or without an implantable defibrillator in advanced chronic heart failure. $N$ Engl J Med. 2004;350(21):2140-2150.

33. Linde C, Abraham WT, Gold MR, et al. Randomized trial of cardiac resynchronization in mildly symptomatic heart failure patients and in asymptomatic patients with left ventricular dysfunction and previous heart failure symptoms. J Am Coll Cardiol. 2008;52(23):1834-1843.

34. Adelstein EC, Saba S. Usefulness of baseline electrocardiographic QRS complex pattern to predict response to cardiac resynchronization. Am J Cardiol. 2009;103(2): 238-242. 